\title{
NON-COLLINEAR MAGNETISM IN ITINERANT-ELECTRON SYSTEMS
}

\author{
J. KÜBLER \\ Darmstadt University of Technology, Institute of Solid State Physics \\ 64289 Darmstadt, Germany
}

\begin{abstract}
The Heisenberg model cannot uncritically be applied to itinerant-electron magnets, including those that show non-collinear order. Density functional theory is therefore generalized to apply to non-collinear itinerant-electron magnets. The appropriate Kohn-Sham equations are discussed and the total energy of spiral magnetic order is used to determine the magnon spectrum in the adiabatic approximation. The energy spectrum of transverse spin fluctuations can also be estimated with the total energy of spiral magnetic order and allows a determination of thermal properties of itinerant-electron magnets. Calculated results for $\mathrm{Fe}, \mathrm{Co}, \mathrm{Ni}$, and $\mathrm{FeCo}$ are discussed and compared with experimental results.
\end{abstract}

PACS numbers: 71.15.Mb, 75.10.Lp, 75.30.Ds, 75.30.Kz

\section{Introduction}

Non-collinear magnetic structures are found among transition-metal systems and the rare earths. They have been discussed in length by e.g. Keffer [1] and Coey [2]. These authors, just as well as others, interpret this physical phenomenon theoretically in the framework of the Heisenberg model assuming localized magnetic moments. While this is certainly justified for the rare-earth systems, for the transition metals and their compounds this approach is questionable because here the magnetic moments originate from itinerant electrons. Collinear magnets made of transition metals have therefore been intensively studied using first-principles energy-band approaches provided by the density functional theory [3, 4]. A good review is that of Staunton [5]. Although non-collinear ground states seemed rather exotic and rare, density functional theoretical energy-band methods were developed and applied to these systems quite successfully in the late eighties [6-8].

Somewhat earlier it became clear that the Stoner theory which explains ground-state properties of the ferromagnetic transition metals quite well, fails to describe the thermal properties of itinerant-electron magnets. The reason for this failure was identified in the neglect of transverse fluctuations of the magnetization that, if viewed as originating from well-developed atomic moments, is equivalent to neglecting fluctuations of the direction of the atomic moments. This thus added 
new incentive to develop non-collinear magnetism further. An extensive discussion of the underlying physics can be found in the paper by Gyorffy et al. [9] and, in a larger context, in the book by Moriya [10].

This is not the place to review all aspects of the presently used first-principles studies of non-collinear magnets. Sandratskii [11] who, in particular, emphasized and unraveled symmetry properties of this phenomenon, went a long way in this direction. Here we want to show that in the adiabatic approximation low lying spin excitations (magnons) and spin fluctuations can be modeled by means of non-collinear magnetic configurations whose total energies may be computed $a b$ initio with only little effort. Previous work along these lines is that by Antropov et al. $[12,13]$ as well as Uhl and Kübler $[14,15]$.

\section{The Kohn-Sham equations}

The derivation of the Kohn-Sham equations for non-collinear magnets follows the standard procedure of density functional theory and was, in fact, already written down by von Barth and Hedin as early as 1972 [16], but was not further pursued at that time. In this case the total energy is considered as a functional of the two-by-two density matrix, $\tilde{n}(\boldsymbol{r})$, and the Kohn-Sham equations are therefore two-component spinor functions [8] whose solutions $\left\{\psi_{i \alpha}(r), i=1, \ldots, \infty\right.$; $\alpha=1,2\}$ define the elements of the density matrix for an $N$-electron system

$$
n_{\beta \alpha}(r)=\sum_{i=1}^{N} \psi_{i \beta}(r) \psi_{i \alpha}^{*}(r),
$$

and determine the density through

$$
n(r)=\operatorname{tr} \tilde{n}(r)
$$

as well as the vector of the magnetization

$$
m(\boldsymbol{r})=\operatorname{tr} \sigma \tilde{n}(r) .
$$

In atomic units the Kohn-Sham equations can be written as

$$
\sum_{\gamma=1}^{2}\left[\delta_{\alpha \gamma}\left(-\nabla^{2}+v\right)+(\boldsymbol{\sigma} \cdot \boldsymbol{B})_{\alpha \gamma}\right] \psi_{i \gamma}=\varepsilon_{i} \psi_{i \alpha},
$$

where $\boldsymbol{B}$ is the effective field written as

$$
\boldsymbol{B}=\left(\sin \theta \cos \varphi \boldsymbol{e}_{x}+\sin \theta \sin \varphi \boldsymbol{e}_{y}+\cos \theta \boldsymbol{e}_{z}\right) B
$$

with $B=B(r)$ given by

$$
B=\frac{1}{2}\left(\frac{\delta E_{\mathrm{xc}}}{\delta n_{1}}-\frac{\delta E_{\mathrm{xc}}}{\delta n_{2}}\right),
$$

where $n_{1}=n_{1}(\boldsymbol{r})$ and $n_{2}=n_{2}(\boldsymbol{r})$ are the local eigenvalues of the spin-density matrix, $E_{\mathrm{xc}}$ is the exchange-correlation energy. Also the polar angles $\theta$ and $\varphi$ depend on $\boldsymbol{r}$.

For practical calculations it is common to use the atomic sphere approximation for the magnetization direction, i.e. the direction of the magnetization is averaged to a constant in the atomic sphere of every atom and is different for different atoms. The vector magnetic moment hence is defined as

$$
M_{n}=\int_{A S(n)} m(r) \mathrm{d} r,
$$


where $A S(n)$ indicates integration over the $n$-th atomic sphere in the crystal. Thus the dependencies on $r$ in Eqs. (1) to (6) are replaced by the coarse grained mesh given by the positions of the atoms.

Further progress is made by an old observation of Herring [17]. Suppose the magnetic moment varies from atom to atom forming a spiral magnetic structure defined by

$$
\boldsymbol{M}_{n}=M\left[\cos \left(\boldsymbol{q} \cdot \boldsymbol{R}_{n}\right) \sin \theta, \sin \left(\boldsymbol{q} \cdot \boldsymbol{R}_{n}\right) \sin \theta, \cos \theta\right],
$$

where the commas separate Cartesian components, $\theta, \varphi=\boldsymbol{q} \cdot \boldsymbol{R}_{n}$ are spherical polar coordinates, and $M$ is the magnitude of the magnetic moments $\boldsymbol{M}_{n}$ (i.e. independent of $n$ ). Then - provided spin-orbit coupling is ignored - it can be shown $[17,11]$ that a combined symmetry operation consisting of a spin rotation through $\varphi=\boldsymbol{q} \cdot \boldsymbol{R}_{n}$ and translation through $\boldsymbol{R}_{n}$, denoted by $\left\{\boldsymbol{q} \cdot \boldsymbol{R}_{n}|\varepsilon| \boldsymbol{R}_{n}\right\}$, commutes with the Kohn-Sham Hamiltonian and allows the formulation of a generalized Bloch theorem of the form

$$
\left\{\boldsymbol{q} \cdot \boldsymbol{R}_{n}|\varepsilon| \boldsymbol{R}_{n}\right\} \psi_{\boldsymbol{k}}(\boldsymbol{r})=\exp \left(-\mathrm{i} \boldsymbol{k} \cdot \boldsymbol{R}_{n}\right) \psi_{k}(r),
$$

where $\psi_{k}(r)$ is the spinor wave function and the vectors $k$ lie in the first Brillouin zone which is defined as usual by the translation vectors $\boldsymbol{R}_{n}$. This form of the Bloch theorem permits to restrict considerations of real space to a chemical unit cell avoiding any supercells. This is an enormous benefit supplied by group theory [11].

The total energy of an itinerant-electron magnet is now calculated by constraining the magnitude of the magnetic moment to a chosen value $M$ and prescribing a value of the spiral $q$-vector as well as the angle $\theta$. The resulting total energy is then

$$
E=E(M, \theta, \boldsymbol{q}) .
$$

Of course, the ground state is given by the total energy minimum. For bcc-Fe, hcp- or fcc-Co, and fcc-Ni this occurs for $q=0$, but, for instance, in fcc-Fe a value of $q=(0,0,0.6) 2 \pi / a$ is found $[18,19]$. Thus, in accordance with experimental evidence, we state that fcc-Fe possesses a non-collinear ground state.

\section{Adiabatic spin dynamics}

If we set out to describe the motion of the magnetization as a function of time at each point $r$ in the crystal, we must determine the equation of motion for $m(r)$. We thus look for the equation of motion of its constituents and are led to the time-dependent Schrödinger (or Kohn-Sham) equation for the wave functions $\psi_{i \alpha}$, which in principle should give the time dependence of the spin-density matrix, $\tilde{n}$, and thus that of $m(r)[12,13]$. Knowing that the band widths of the $d$-electrons in the crystal are of the order of electron volts, while the low-lying excitations we look for are spin-waves which have energies of the order of milli-electron volts, we may assume that the motion of the electrons is much faster than that of the magnetic moments. This assumption implies that we imagine different timescales govern the physics of the electrons and the magnetic moments [9]. The problem is comparable to the Born-Oppenheimer or adiabatic approximation with which we separate the motion of the electrons from that of the nuclei. It has become common to use the term adiabatic here. Nevertheless, it is important to emphasize that a 
systematic adiabatic approach has not been developed for the spin problem, simply because - in contrast to the nuclear motion - there is no large mass governing the timescale. The approach to take is to carry out the necessary calculations and compare the results with experimental data which will decide whether or not the approximations are tolerable.

In the adiabatic approximation one assumes that the time-dependent Schrödinger equation holds at any instant of time for a potential that parametrically depends on time. The energy eigenvalues are then supposed to depend on time in such a way that no levels cross and transitions to other levels do not occur.

In our case the time-dependent parameters in the Schrödinger equation are the angles $\theta$ and $\phi$ in Eq. (8). The essential assumption now is that the time-dependent Schrödinger equation holds in the context of density-functional theory in the adiabatic approximation. It has been shown by Gross et al. [20] that density-functional theory of time-dependent phenomena can be justified extremely well, so that we can work out the equation of motion for the magnetization by evaluating $\partial m(r) / \partial t$ using Eq. (3) etc. These equations are subsequently linearized and one finds for the magnon frequency in the case of elementary ferromagnets

$$
\omega(\boldsymbol{q})=\lim _{\theta \rightarrow 0} \frac{4}{M} \frac{\Delta E(\boldsymbol{q}, \theta)}{\sin ^{2} \theta} .
$$

Here $\Delta E(q, \theta)$ is the total energy difference with respect to the ground state. Details of the derivation will appear elsewhere. Using completely different methods this result has been written down before by other authors [21-23].

For small $\boldsymbol{q}$ we calculate the spin-wave stiffness constant by means of

$$
\omega(q)=D|q|^{2}\left(1-\beta|q|^{2}\right)
$$

and compare the results for the elementary ferromagnets in Table I with the calculation of Rosengaard and Johansson [21] and with experimental results.

Calculated magnon spectra for the elementary ferromagnets using $\boldsymbol{q}$-vectors in the entire Brillouin zone can be found in the paper by Halilov et al. [22] where they are also, with good success, compared with experimental data. We here show

\section{TABLE I}

Spin-wave stiffness constants, $D$, in $\mathrm{meV} \AA^{-2}$. Experimental data points shown were obtained by Tajima et al. [24] at $4.2 \mathrm{~K}$.

\begin{tabular}{l|c|c|c}
\hline \hline & Calc. $^{a}$ & Calc. $^{b}$ & Exp. \\
\hline bcc-Fe & 247 & 355 & 314 \\
fcc-Co & 502 & 535 & 510 \\
fcc-Ni & 739 & 790 & 550 \\
\hline
\end{tabular}




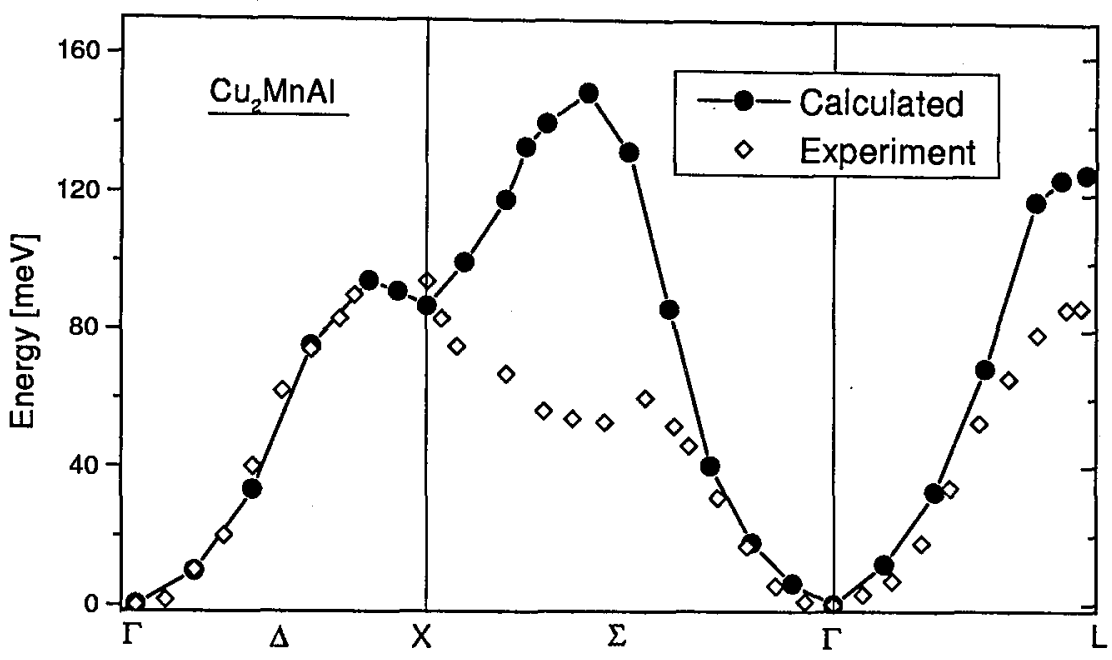

Fig. 1. Adiabatic magnon-dispersion relations along high-symmetry lines of $\mathrm{Cu}_{2} \mathrm{MnAl}$.

results for a prominent Heusler alloy, $\mathrm{Cu}_{2} \mathrm{MnAl}$. Although this is obviously not elementary and in the literature is considered to be an "ideal local moment system" we calculated the magnon spectrum using Eq. (11) by freezing the small induced moments at the $\mathrm{Cu}$ - and $\mathrm{Al}$-sites to zero. The results are shown in Fig. 1. The agreement with the experimental data is only moderate with rather large deviations along the $\Sigma$-axis. The reason for this discrepancy is not known at present.

\section{Spin fluctuations and the Curie temperature}

At high temperatures the spin waves begin to interact and the magnon picture is to be replaced by a picture of strong long-wavelength transverse spin fluctuation. This is not the place to present a full derivation of the thermal properties near the Curie temperature, $T_{C}$, which has been given in Refs. $[14,15,26]$. The salient features, however, can be described as follows.

First, the total energy differences from spiral magnetic states are written as

$$
\Delta E(M, \boldsymbol{q}, \theta)=\sum_{n=1}^{n_{m}} \alpha_{2 n} M^{2 n}+\sum_{n=0}^{n_{m}-1} j_{n}(\boldsymbol{q}) M^{2 n+2} \sin ^{2} \theta .
$$

Fitting the total energy differences of the type shown as an example in Fig. 2 supplies the constants $\alpha_{2}, \alpha_{4}, \ldots$ and the exchange functions $j_{0}(q), j_{1}(q), \ldots$ of which only those written out are needed in first approximation. Next, the magnetization is expanded in terms of fluctuations $m_{j} k$ where $j$ labels Cartesian coordinates,

$$
\boldsymbol{M}(\boldsymbol{R})=M \boldsymbol{e}_{z}+\boldsymbol{m}(\boldsymbol{R})=M \boldsymbol{e}_{z}+\sum_{j, \boldsymbol{k}} \exp (\mathrm{i} \boldsymbol{k} \cdot \boldsymbol{R}) \boldsymbol{e}_{j}
$$

$\boldsymbol{R}$ being translation vectors. A Hamilton function in terms of the fluctuation parameters is then constructed whose $T=0$ average gives the total energy differences, Eq. (13). Using the Bogolyubov-Peierls variational principle and a Gaussian model Hamiltonian we can then determine the Helmholtz free energy which 


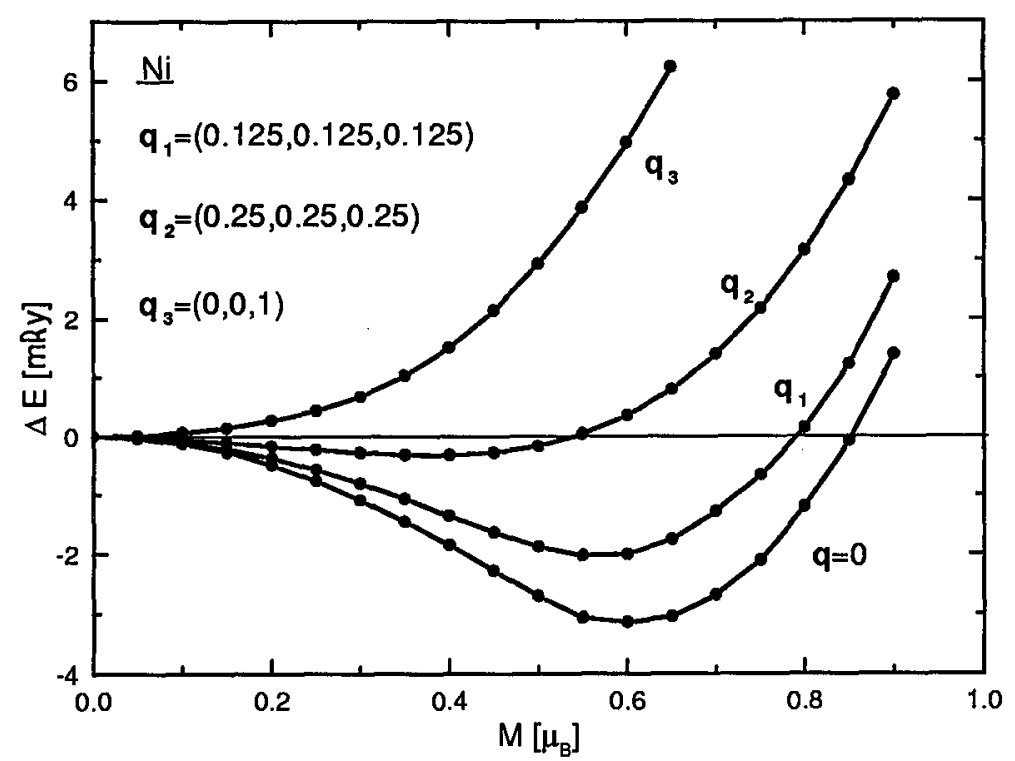

Fig. 2. Total-energy differences for $\mathrm{Ni}$ as function of the magnetization, $M$, for several values of the spiral vector $\boldsymbol{q}$ (given in units of $2 \pi / a$ ). Polar angle $\theta=90^{\circ}$.

TABLE II

Calculated magnetic properties of the elementary ferromagnets, compared with experimental data. $T_{\mathrm{C}}$ - Curie temperature, $M_{\mathrm{s}}$ - magnetic moment at $T=0, q_{\mathrm{c}}$ - "number of magnetic carriers", $E_{\mathrm{s}}$ - energy gain in magnetic state, $\sum_{q} \Delta E\left(M_{\mathrm{s}}, \boldsymbol{q}, \theta=\right.$ $\pi / 2$ ) - Brillouin-zone sum over total-energy differences.

\begin{tabular}{lr|c|c|c|c|c}
\hline \hline & & bcc-Fe & fcc-Co & bcc-Co & hcp-Co & fcc-Ni \\
\hline$T_{\mathrm{C}}[\mathrm{K}]$ & calc. $^{a}$ & 1180 & 950 & 1205 & 1076 & 370 \\
& calc. $^{b}$ & 1095 & 1012 & & & 412 \\
& exp. & 1044 & 1388 & & & 627 \\
\hline$M_{\mathrm{s}}\left[\mu_{\mathrm{B}}\right]$ & calc. & 2.20 & 1.61 & 1.69 & 1.60 & 0.62 \\
& exp. & 2.216 & & $1.7^{c}$ & 1.715 & 0.616 \\
\hline$q_{\mathrm{c}}$ & calc. & 1.40 & 1.12 & 1.07 & & 0.28 \\
& exp. & 2.29 & 2.29 & & & 0.9 \\
\hline$E_{\mathrm{s}}[\mathrm{mRy}]$ & 26.0 & 10.2 & 17.1 & 10.8 & 3.4 \\
\hline$\sum_{q} \Delta E\left(M_{\mathbf{s}}, \boldsymbol{q}, \theta=\pi / 2\right)[\mathrm{mRy}]$ & 12.5 & 14.8 & 16.8 & 12.8 & 6.1 \\
\hline
\end{tabular}

${ }^{a}$ Results obtained with $n_{m}=2 ;{ }^{b}$ Higher order corrections, $n_{m}>2$, employed;

${ }^{c}$ Bland et al. [25].

supplies all thermal properties. We collect in Table II the results for elementary ferromagnets. It is seen that generally the Curie temperatures of the fcc systems are underestimated, while those of the bcc systems are overestimated. Empirically, 
it is seen that the quantity $\sum_{q} \Delta E\left(M_{\mathrm{s}}, \boldsymbol{q}, \theta=\pi / 2\right)$ listed in Table II reproduces the trend in the experimental Curie temperatures. The so-called number of magnetic carriers, $q_{\mathrm{c}}$ is defined by means of the Curie constant which is written as $C=(1 / 3) q_{\mathrm{c}}\left(q_{\mathrm{c}}+2\right) \mu_{\mathrm{B}}^{2} / k_{\mathrm{B}}$, where $k_{\mathrm{B}}$ is Boltzmann's constant. Thus the Curie constant is generally underestimated.

In Fig. 3 we show an example of $a b$ initio computed thermal properties. We have chosen here bcc-Co which is not the stable form, but is experimentally produced by thin-film techniques. We thus predict the Curie temperature of assumed bulk bcc-Co which, most likely, is an overestimate.

The fluctuations $t^{2}, l^{2}$, and $p^{2}$ depicted in Fig. 3 are defined as

$$
t^{2}=\left(1 / M^{2}\right) \sum_{k}\left\langle\left|m_{t k}\right|^{2}\right\rangle_{0} \quad \text { and } \quad l^{2}=\left(1 / M^{2}\right) \sum_{k}\left\langle\left|m_{l k}\right|^{2}\right\rangle_{0}
$$

where $t$ and $l$ denote the transverse and longitudinal Cartesian coordinates, respectively. In the paramagnetic state $t^{2}$ and $l^{2}$ are degenerate and are, therefore, denoted by $p^{2}=t^{2}=l^{2}$. Furthermore, we notice from the inset in Fig. 3 that the phase transition at the Curie temperature is of first order. This is a defect of the theory which must be removed in the future. Also notice that the inverse paramagnetic susceptibility is Curie-Weiss like, as it should be: a serious defect of the old Stoner theory for band magnetism which predicted a Pauli-like susceptibility has disappeared.

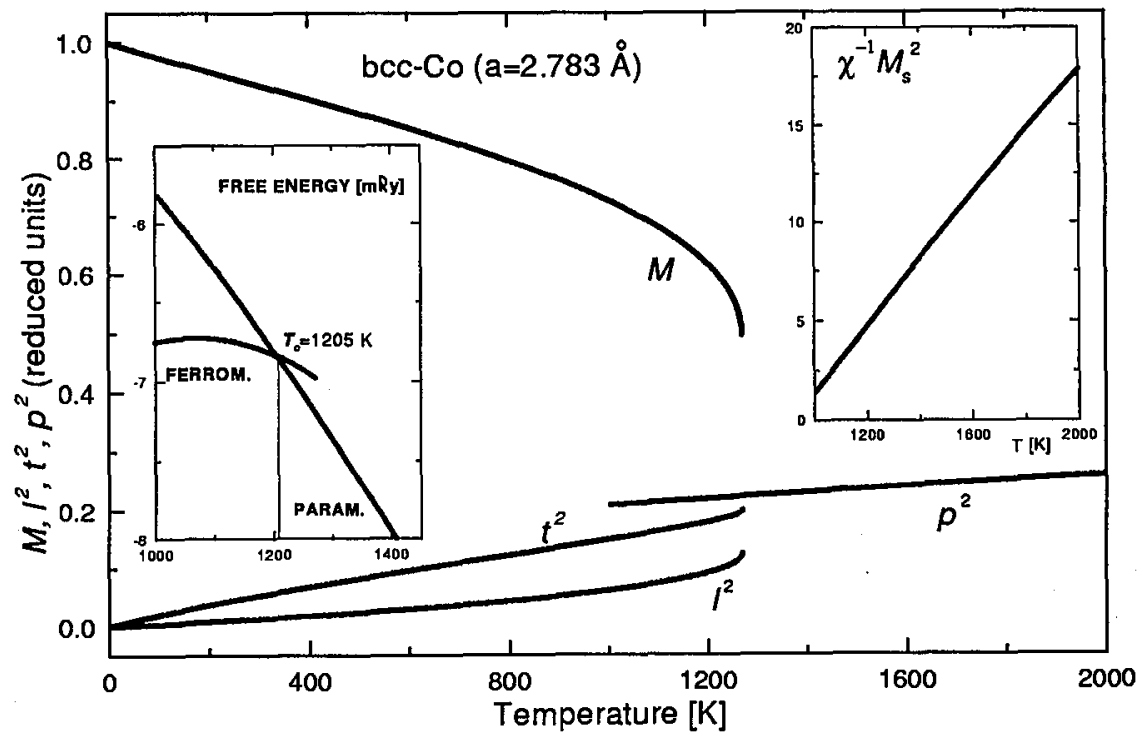

Fig. 3. Calculated magnetization data for bcc-Co as functions of temperature. $M$ is the reduced magnetization, $l^{2}, t^{2}$, and $p^{2}$ are the reduced fluctuations defined by Eq. (15). Insets: free energy for the ferromagnetic and paramagnetic cases (temperature given is the calculated Curie temperature); inverse paramagnetic susceptibility multiplied with the saturation magnetization squared, $M_{\mathrm{s}}^{2}$, in mRy. 


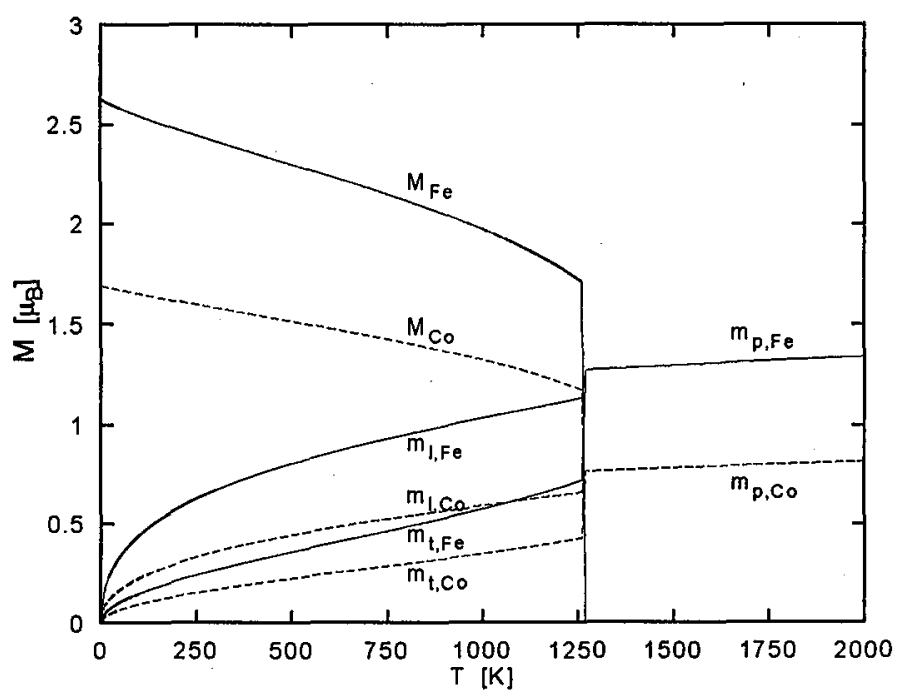

Fig. 4. Calculated magnetization $(M)$ and the transverse, longitudinal, and paramagnetic fluctuations at the $\mathrm{Fe}$ and $\mathrm{Co}$ sites in $\mathrm{FeCo}$. (From unpublished calculations of M. Uhl.) Here, with Eq. (15), $m_{t, \mathrm{Fe}}=M_{\mathrm{Fe}} \sqrt{t_{\mathrm{Fe}}^{2}}, m_{l, \mathrm{Fe}}=M_{\mathrm{Fe}} \sqrt{l_{\mathrm{Fe}}^{2}}, m_{p, \mathrm{Fe}}=M_{\mathrm{Fe}} \sqrt{p_{\mathrm{Fe}}^{2}}$ etc. for Co. The experimental Curie temperature is $1235 \mathrm{~K}$.

Of basic and technological interest are predictions of the magnetic properties of compounds and artificial structures like for instance multilayers. It is thus important to extend spin-fluctuation theory to these cases as well. This turns out to be somewhat more involved since the constrained total energy is now a function of many more variables than for the case of elementary systems. For example, the total energy can be constrained separately for each constituent atom which possess separate spin fluctuations. The problem appears roughly like in the theory of phonons for non-elementary systems where a secular equation determines the various frequency branches. As an example we show in Fig. 4 the calculated magnetization and the fluctuations for the compound $\mathrm{FeCo}$. Certainly, the order of the transition is reproduced incorrectly, but the calculated Curie temperature is very close to the experimental value (which could be fortuitous). Details of the theory will be given elsewhere.

\section{Acknowledgment} Mainz.

This work was supported by the DFG: SFB 252 Darmstadt, Frankfurt,

\section{References}

[1] F. Keffer, in: Encyclopedia of Physics, Vol. 18/2, Ed. H.P.J. Wijn, Springer-Verlag, Berlin 1966, p. 1.

[2] J.M.D. Coey, Can. J. Phys. 65, 1210 (1987).

[3] P. Hohenberg, W. Kohn, Phys. Rev. B 136, 864 (1964).

[4] W. Kohn, L.J. Sham, Phys. Rev. A 140, 1133 (1965). 
[5] J.B. Staunton, Rep. Prog. Phys. 57, 1289 (1994).

[6] J. Kübler, K.-H. Höck, J. Sticht, A.R. Williams, J. Phys. F, Metal Phys. 12, 469 (1988).

[7] J. Kübler, K.-H. Höck, J. Sticht, A.R. Williams, J. Appl. Phys. 63, 3482 (1988).

[8] J. Sticht, K.-H. Höck, J. Kübler, J. Phys., Condens. Matter 1, 8155 (1989).

[9] B.L. Gyorffy, A.J. Pindor, J. Staunton, G.M. Stocks, H. Winter, J. Phys. F, Metal Phys. 15, 1337 (1985).

[10] T. Moriya, Spin Fluctuations in Itinerant Electron Magnetism, Springer-Verlag, Berlin 1985, p. 1.

[11] L.M. Sandratskii, Adv. Phys. 47, 91 (1998).

[12] V.P. Antropov, M.I. Katsnelson, M. van Schilfgaarde, B.N. Harmon, Phys. Rev. Lett. 75, 729 (1995).

[13] V.P. Antropov, M.I. Katsnelson, M. van Schilfgaarde, B.N. Harmon, D. Kuznezov, Phys. Rev. B 54, 1019 (1996).

[14] M. Uhl, J. Kübler, Phys. Rev. Lett. 77, 334 (1996).

[15] M. Uhl, J. Kübler, J. Phys., Condens. Matter 9, 7885 (1997).

[16] U. von Barth, L. Hedin, J. Phys. C 5, 1629 (1972).

[17] C. Herring, in: Magnetism IV, Eds. G. Rado, H. Suhl, Academic Press, New York 1966, Chaps. V, XIII.

[18] O.N. Mryasow, A.I. Liechtenstein, L.M. Sandratskii, V.A. Gubanov, J. Phys., Condens. Matter 3, 7683 (1991).

[19] M. Uhl, L.M. Sandratskii, J. Kübler, J. Magn. Magn. Mater. 103, 314 (1992).

[20] E.K.U. Gross, J.F. Dobson, M. Petersilka, in: Density Functional Theory, Ed. R.F. Nalewajski, Springer-Verlag, Berlin 1996, p. 1.

[21] N.M. Rosengaard, B. Johansson, Phys. Rev. B 55, 14975 (1997).

[22] S.V. Halilov, H. Eschrig, A.Y. Perlov, P.M. Oppeneer, Phys. Rev. B 58, 293 (1998).

[23] Q. Niu, L. Kleinman, Phys. Rev. Lett. 80, 2205 (1998).

[24] K. Tajima, Y. Ishikawa, P.J. Webster, M.W. Stringfellow, D. Tocchetti, K.R.A. Zeabeck, J. Phys. Soc. Jpn. 43, 483 (1977).

[25] J.A.C. Bland, R.D. Bateson, P.C. Riedi, R.G. Graham, H.J. Lauter, J. Penfold, C. Shackleton, J. Appl. Phys. 69, 4989 (1991).

[26] L.M. Sandratskii, M. Uhl, J. Kübler, in: Itinerant Electron Magnetism: Fluctuation Effects, Ed. D. Wagner, W. Brauneck, A. Solontsov, Kluwer Academic Publ., Dordrecht 1998, p. 161. 